\title{
ANALYTIC DISTRIBUTION KERNELS
}

\section{BY \\ J. BARROS NETO(' $\left.{ }^{1}\right)$}

Introduction. In his study of the structure of distribution kernels (centering around his celebrated kernel theorem), L. Schwartz $[11 ; 13]$ has studied the subclasses of regular and very regular distribution kernels, which may be characterized roughly by the fact that they carry infinitely differentiable functions into infinitely differentiable functions. It is our object in the present paper to extend this study to kernels which are analytically regular, i.e., which, again roughly, carry analytic functions into analytic functions. The motivation for such a study is provided by the fundamental solutions of elliptic equations with analytic coefficients. One consequence of our results is that for such equations the standard theorems on the analyticity of regular solutions imply the analyticity of the fundamental solution.

The principal tool in our investigation is the general theory of topological vector spaces (particularly in certain forms given to it by A. Grothendieck) and especially the theory of topological tensor products. In $\S I .1$, we summarize the definitions from the theory of topological vector spaces which we shall use. In $\$ I .2$, we give a brief summary of the definitions and principal results of Grothendieck'stheory of topological tensor products. In §I.3, we summarize the results of Schwartz's study of distribution kernels, at least insofar as they relate to the generalizations to be given to analytic kernels. §II is devoted to an intensive consideration of the space $a(K)$ of real analytic functions on a compact subset of $R^{n}$. §III states and proves our principal results on analytic distribution kernels.

The writer should like to thank Professors L. Nachbin and F. E. Browder for discussion, suggestions, and criticism. The results of the present paper constitute a portion of the writer's Doctoral Dissertation [1] at the University of São Paulo, prepared while in residence at the Institute for Pure and Applied Mathematics, Rio de Janeiro.

\section{Preliminaries}

1. Topological vector spaces. We shall follow the definitions and notations of the treatment of topological vector spaces given by Bourbaki [3] and Grothendieck [8].

Let $E$ be a topological vector space of $R$, the field of the real numbers, or $C$, the field of the complex numbers.

A subset $A$ of $E$ is called circled if $\lambda A \subset A$ for all scalars $\lambda$ such that $|\lambda| \leqq 1$.

Received by the editors January 19, 1961.

(1) Fellow from Comissão Nacional de Energia Nuclear, Brazil. 
We say that $A$ absorbs $B$ if there exists a real number $\epsilon>0$ such that for all $\lambda$ with $|\lambda|<\epsilon$, we have $\lambda B \subset A$. The subset $A$ is called absorbing if it absorbs every $x \in E$. $A$ is called a bounded set if every neighborhood of zero in $E$ absorbs $A$.

We say that $A$ is a convex set if, for all $x, y \in A, \lambda x+(1-\lambda) y \in A$, for all $\lambda$ such that $0<\lambda<1$.

By the circled convex hull of $A, \Gamma(A)$, we mean the smallest circled convex set which contains $A . \Gamma(A)$ is, obviously, the set of all finite sums $\sum \lambda_{i} x_{i}$, with $x_{i} \in A$ and the scalars $\lambda_{i}$ such that $\sum\left|\lambda_{i}\right| \leqq 1$.

The topological vector space $E$ is called locally convex if its topology admits a fundamental system of neighborhoods of zero consisting of convex, circled and absorbing sets. In this case the topology of $E$ can be defined by a family of semi-norms. All the spaces to be considered will be locally convex and separated.

Let $E^{\prime}$ be the dual space of $E$, i.e., the space of all continuous linear functionals on $E$. Let $A$ be a subset of $E$; the polar of $A$ is the set of all elements $x^{\prime} \in E^{\prime}$ such that:

$$
\left|\left\langle x, x^{\prime}\right\rangle\right| \leqq 1
$$

for all $x \in A$, where $\langle$,$\rangle denotes the pairing between E$ and $E^{\prime}$. We denote by $A^{0}$ the polar of $A$. An analogous definition may be given for the subsets of $E^{\prime}$.

Let $\subseteq$ be a set of bounded subsets of $E$; the $\Im$-convergence topology on $E^{\prime}$ is defined by taking as the fundamental system of neighborhoods of zero, all the finite intersections of non-null homothetics of polars of elements of $\mathfrak{S}$.

If $\subseteq$ is the set of all finite subsets of $E$ (resp. $E^{\prime}$ ) the $\subseteq$-convergence topology on $E^{\prime}$ (resp. $E$ ) is the weak topology of $E^{\prime}$ (resp. $E$ ). We denote by $s\left(E^{\prime}, E\right)$ (resp. $s\left(E, E^{\prime}\right)$ ) this topology and by $E_{s}$ (resp. $E_{s}^{\prime}$ ) the space $E$ (resp. $E^{\prime}$ ) with the weak topology. If $\subseteq$ is the set of all bounded sets of $E$, the $\subseteq$-convergence topology on $E^{\prime}$ is the strong topology.

A locally convex topological vector space $E$ is said to be reflexive (resp. a Montel space) if every bounded subset is weakly relatively compact (resp. relatively compact).

A Fréchet space, (F)-space, is a locally convex topological vector space which is metrisable and complete.

The space $E$ is called almost-complete if every bounded closed subset of $E$ is complete. Every complete space is, obviously, almost-complete.

If $M$ is an equicontinuous subset of $E^{\prime}$ (i.e. $M$ is the polar of a neighborhood of zero in $E$ ), $M$ is bounded in every 5 -convergence topology on $E^{\prime}$, where $\subseteq$ is any family of bounded subsets of $E$. In particular, $M$ is weakly bounded and strongly bounded.

The space $E$ is called almost-barrelled if every strongly bounded subset $M$ of $E^{\prime}$ is equicontinuous. $E$ is called barrelled if any weakly bounded subset $M$ of $E^{\prime}$ is equicontinuous. All barrelled spaces are almost-barrelled. 
$E$ is called a bornological space if every convex circled subset of $E$ which absorbs the bounded subsets of $E$ is a neighborhood of zero in $E$. If $E$ is bornological, every linear mapping from $E$ into a locally convex space $F$ which maps the bounded sets of $E$ into bounded sets of $F$ is continuous [8, p. 200, Definition 4 and Proposition 6].

Let $A$ be a convex, bounded and circled subset of $E$; by $E_{A}$ we mean the vector subspace of $E$ spanned by $A$, with the topology defined by the seminorm:

$$
\|x\|=\inf \{|\lambda|: x \in \lambda A\} .
$$

If in addition $A$ is complete, then $E_{A}$ is a Banach space [8, p. 190, Lemma 1]. It is obvious that the topology on $E_{A}$ induced by the containing space $E$ is weaker than the topology of $E_{A}$.

Let $\left(E_{i}\right)_{i \in I}$ be a family of locally convex spaces, $E$ a vector space, $\left(u_{i}\right)_{i \in I}$ a family of mappings of $E_{i}$ into $E$ such that the union of the images $u_{i}\left(E_{i}\right)$ is $E$. The inductive limit topology on $E$ is defined as the strongest of the locally convex topologies on $E$ for which the mappings $u_{i}$ are continuous. A fundamental set of neighborhoods of zero is obtained by taking all convex and circled subsets $V$ of $E$ such that $u_{i}^{-1}(V)$ is a neighborhood of zero on $E_{i}$, for all $i \in I$.

It is always possible to restrict this general definition of inductive limit topology to the case where the spaces $E_{i}$ are subspaces of $E$ the union of which is $E$, the index set $I$ is a directed set, and if $E_{i} \subset E_{j}$ the identity mapping from $E_{i}$ into $E_{j}$ is continuous. It is obvious that if $E_{i} \subset E$ the topology of $E_{i}$ is stronger than the induced topology of $E$.

It is easy to show that if the spaces $E_{i}$ are barrelled (resp. almost-barrelled, resp. bornological) then $E$ is barrelled (resp. almost-barrelled, resp. bornological).

By a generalized (LF)-space we mean a locally convex separated space $E$ which is the inductive limit of a sequence $\left(E_{i}\right)$ of $(\mathcal{F})$-spaces by linear mappings $u_{i}$. We may always suppose that $\left(E_{i}\right)$ is an increasing sequence of $(\mathcal{F})$-spaces contained in $E$ such that its union is $E$ and such that the identity mapping from $E_{i}$ into $E_{i+1}$ is continuous.

The definition of (\&F)-space given by Dieudonné and Schwartz in [6] corresponds to the special case in which the induced topology of $E_{i+1}$ on $E_{i}$ coincides with the topology of $E_{i}$. For such spaces, one may prove that $E$ induces on $E_{i}$ the topology of $E_{i}$; if the spaces $E_{i}$ are complete, it follows that $E$ is complete and every bounded subset of $E$ is contained and bounded in some $E_{i}$. These conclusions are not known for generalized (LF)-spaces. In $\S I I$, however, we shall consider a case in which these last two conclusions are verified for a generalized (LF)-space which is not an $(\mathscr{L F})$-space in the sense of [6]. 
2. Topological tensor products (Grothendieck [7], Schwartz [12]).

Let $E$ and $F$ be two locally convex separated spaces and let $E \otimes F$ be the tensor product of these spaces. It is always possible to define on $E \otimes F$ various topologies which agree with the vector space structure on $E \otimes F$. We are interested in only two of these, namely, the $\pi$-topology and the $\epsilon$-topology whose principal properties we give, briefly, below.

There is a unique locally convex separated topology on $E \otimes F$ such that for every locally convex space $G$, the space $L(E \otimes F, G)$ of all continuous linear mappings from $E \otimes F$ into $G$, coincides with the space $B(E, F ; G)$ of continuous bilinear mappings from $E \times F$ into $G$. In this identification, to an equicontinuous subset of $L(E \otimes F, G)$ there corresponds an equicontinuous subset of $B(E, F ; G)$ and conversely. In particular, the dual space of $E \otimes F$ coincides with $B(E, F)$, the space of all bilinear continuous functionals on $E \times F$. We denote by $\pi$ this topology, by $E \otimes_{\pi} F$ the space $E \otimes F$ with the $\pi$-topology and by $E \hat{\otimes}_{\pi} F$ its completion. A fundamental system of neighborhoods of zero in the $\pi$-topology is obtained by taking the circled convex hulls, $\Gamma(U \otimes V)$, of $U \otimes V$, where $U$ (resp. $V$ ) runs through a fundamental system of neighborhoods of zero in $E$ (resp. $F$ ). The $\pi$-topology is the strongest one on $E \otimes F$ for which the canonical mapping $(x, y) \in E \times F \rightarrow x \otimes y \in E \otimes F$ is continuous.

Let $\mathfrak{B}\left(E_{s}^{\prime}, F_{s}^{\prime}\right)$ be the space of bilinear functionals continuous in each variable separately on $E_{s}^{\prime} \times F_{s}^{\prime}$. This space coincides with the space $L\left(F_{s}^{\prime}, E_{s}\right)$ of linear continuous mappings from $F_{s}^{\prime}$ into $E_{s}$ and also with the space $L\left(E_{s}^{\prime}, F_{s}\right)$. Let us denote by $E_{t}$ (resp. $\left.E_{t}^{\prime}\right)$ the space $E$ (resp. $E^{\prime}$ ) with the Mackey topology $t\left(E, E^{\prime}\right)$ (resp. $t\left(E^{\prime}, E\right)$ ) of uniform convergence on the convex circled weakly compact sets of $E^{\prime}$ (resp. $E$ ). It is well known that $L\left(F_{s}^{\prime}, E_{s}\right)$ coincides with the space $L\left(F_{t}^{\prime}, E\right)$ of linear continuous mappings from $F_{t}^{\prime}$ into $E$ [8, p. 151, Corollary 2]. In addition, if $\mathfrak{B}_{e}\left(E_{s}^{\prime}, F_{s}^{\prime}\right)$ denotes $\mathfrak{B}\left(E_{s}^{\prime}, F_{s}^{\prime}\right)$ with the topology of the uniform convergence on the product of all equicontinuous sets of $E^{\prime}$ by all equicontinuous sets of $F^{\prime}$ and if $L_{\varepsilon}\left(F_{\ell}^{\prime}, E\right)$ denotes $L\left(F_{t}^{\prime}, E\right)$ with the topology of the uniform convergence on the equicontinuous sets of $F^{\prime}$, then we have the identities:

$$
\mathfrak{B}_{\theta}\left(E_{s}^{\prime}, F_{s}^{\prime}\right)=L_{\theta}\left(F_{t}^{\prime}, E\right)=L_{\theta}\left(E_{t}^{\prime}, F\right) .
$$

It is easy to see that $E \otimes F$ can be identified to a subspace of $\mathfrak{B}\left(E_{s}^{\prime}, F_{s}^{\prime}\right)$. We denote by $E \otimes_{\epsilon} F$ the space $E \otimes F$ with the induced topology of $\mathfrak{B}_{e}\left(E_{s}^{\prime}, F_{s}^{\prime}\right)$ and by $E \hat{\otimes}_{\epsilon} F$ its completion. When $E$ and $F$ are complete spaces, then $\mathscr{B}_{e}\left(E_{s}^{\prime}, F_{s}^{\prime}\right)$ is complete; hence, $E \hat{\otimes}_{e} F$ may be considered as a subspace of the preceding one. The canonical bilinear mapping $(x, y) \rightarrow x \otimes y$ from $E \times F$ into $E \otimes_{\mathrm{G}} F$ is continuous, which proves that the $\pi$-topology is stronger than the $\epsilon$-topology. These two topologies do not coincide in general.

A separated locally convex space $E$ is said to be nuclear if $E \otimes_{\boldsymbol{\epsilon}} F=E \otimes_{\mathbf{\pi}} F$ for all separated locally convex spaces $F$. The spaces $\mathcal{D}(V), \mathcal{E}(V), \mathcal{E}^{\prime}(V)$ and 
$D^{\prime}(V)$ of the infinitely differentiable functions with compact support, infinitely differentiable functions, distributions with compact support and distributions, respectively, on a differentiable manifold $V$ are nuclear [7, Chapter 2, $\$ 2$, No. 3, Theorem 10]. If $V$ is a complex manifold, the space $\operatorname{FC}(V)$ of holomorphic functions on $V$ is nuclear $[7$, Chapter $2, \S 2$, Theorem 10, corollary].

If $E$ is a separated locally convex space, let us denote by $E_{c}^{\prime}$ the dual space of $E$ with the topology of the uniform convergence on the compact sets of $E . E_{c}^{\prime}$ is stronger than $E_{s}^{\prime}$, and weaker than $E_{t}^{\prime}$; hence the dual space of $E_{c}^{\prime}$ is $E$. It is well known that $L\left(F_{c}^{\prime}, E\right)$ can be identified with the linear subspace of $L\left(F_{s}^{\prime}, E_{s}\right.$ ) (hence of $L\left(F_{t}^{\prime}, E\right)$ ) of all continuous mappings from $F_{\text {? }}^{\prime}$ into $E_{\text {s }}$ which transform the equicontinuous sets of $F^{\prime}$ into relatively compact sets of $E$ [12, exposé 8, Proposition 4].

On the other hand, $E \otimes F$ can be identified to the subspace of $L\left(F_{c}^{\prime}, E\right)$ of linear continuous mappings from $F_{c}^{\prime}$ into $E$ of finite range and then $E \otimes_{\epsilon} F$ will be a subspace of $L_{e}\left(F_{c}^{\prime}, E\right)$. If $E$ and $F$ are complete, $L_{e}\left(F_{c}^{\prime}, E\right)$ is complete $[12$, exposé 8 , Proposition 5$]$; hence, $E \hat{\otimes}_{\bullet} F$ can be identified with a linear subspace of $L_{e}\left(F_{c}^{\prime}, E\right)$. It is obvious that $E \hat{\otimes}_{\epsilon} F$ coincides with $L_{e}\left(F_{c}^{\prime}, E\right)$ if and only if $E \otimes F$ is dense in $L_{e}\left(F_{c}^{\prime}, E\right)$.

We say that a separated locally convex space $F$ verifies the approximation property if the subspace $E \otimes E^{\prime}$ of $L(E, E)$ of mappings with finite-dimensional range is dense in $L(E, E)$ with respect to the topology of the uniform convergence on the convex compact sets of $E$. A separated locally convex space $E$ verifies the approximation property if and only if, for every locally convex space $F, E \otimes F$ is dense in $L_{\theta}\left(F_{c}^{\prime}, E\right)$ [12, exposé 14 , Theorem 2]. If $E$ and $F$ are complete and if $E$ verifies the approximation property, then $E \hat{\otimes}_{c} F=L_{\bullet}\left(F_{c}^{\prime}, E\right)$.

The spaces $D(V), \mathcal{E}(V), \mathcal{E}^{\prime}(V), D^{\prime}(V)$ and $\mathcal{H}(V)$ verify the approximation property.

If $E$ is a complete separated locally convex space, the space $\varepsilon(V, E)$ of infinitely differentiable functions defined on $V$ with values on $E$ with the topology of the uniform convergence on compact subsets of the function and all its derivatives can be identified with $\mathcal{E}(V) \hat{\otimes}_{\pi} E[7$, Chapter II, p. 81, example 1]. Since $\mathcal{E}(V)$ is nuclear (hence verifies the approximation property [12, exposé 17, Proposition 4]) we have:

$$
\varepsilon(V, E)=\varepsilon(V) \hat{\otimes} E=L_{\bullet}\left(E_{c}^{\prime}, \varepsilon(V)\right) .
$$

In particular, if $E=\varepsilon(W)$ we have:

$$
\varepsilon(V \times W)=\varepsilon(V, \mathcal{E}(W))=\varepsilon(V) \hat{\otimes} \mathcal{E}(W)=L_{\epsilon}\left(\mathcal{E}^{\prime}(W), \varepsilon(V)\right) .
$$

In the same way

$$
\mathfrak{H C}(V \times W)=\mathfrak{H C}(V, \mathfrak{H C}(W))=\mathfrak{H C}(V) \hat{\otimes} \mathfrak{H C}(W)=L_{\bullet}\left(\mathfrak{F C}^{\prime}(W), \mathfrak{F C}(V)\right) .
$$


In §II we discuss an analogous situation for the spaces of analytic functions on compact subsets of a real analytic manifold.

3. Distribution kernels. Let $V$ be an $n$-dimensional orientable differentiable manifold, $W$ another such manifold of dimension $m$. Let $D(V \times W)$ and $\mathcal{E}(V \times W)$ be, respectively, the corresponding spaces of infinitely differentiable functions on $V \times W$; let $\mathbb{D}^{\prime}(V \times W)$ and $\mathcal{E}^{\prime}(V \times W)$ be the space of distributions and of distributions with compact support. We use the topologies on these spaces defined by L. Schwartz in [10]. We remark that, since there exists on $V$ an infinitely differentiable $n$-form $\omega$, different from zero, the mapping $f \in \mathcal{E}(V) \rightarrow f \cdot \omega \in \mathscr{D}^{\prime}(V)$ gives an imbedding of $\mathcal{E}(V)$ into $\mathscr{D}^{\prime}(V)$, two of such imbeddings being isomorphic. (If $V$ is an analytic manifold, $\omega$ may be chosen analytic by the imbedding theorem of Morrey and Grauert and the family of analytic distributions is also uniquely defined.)

DEFINITION 1. The elements of $D^{\prime}(V \times W)$ will be called distribution kernels or kernels on $V \times W$.

To a given distribution kernel $K_{x, y}$ there corresponds: (1) a linear continuous map $L_{K}$ from $\mathfrak{D}(W)$ into $\mathfrak{D}^{\prime}(V)$ defined by

$$
\left\langle L_{K}(g), f\right\rangle=\left\langle K_{x, y}, f(x) \otimes g(y)\right\rangle
$$

for all $f \in \mathscr{D}(V)$ and all $g \in \mathscr{D}(W)$; (2) a linear continuous map ${ }^{t} L_{K}$ from $\mathscr{D}(V)$ into $\mathbb{D}^{\prime}(W)$ (the transpose of the preceding one) defined by

$$
\left\langle{ }^{t} L_{K}(f), g\right\rangle=\left\langle K_{x, y}, f(x) \otimes g(y)\right\rangle
$$

for all $f \in \mathscr{D}(V)$ and all $g \in \mathscr{D}(W)$.

We have remarked that $D^{\prime}(V)$ is a nuclear space. Since it is complete we have

$$
\mathscr{D}^{\prime}(V) \hat{\otimes} \mathscr{D}^{\prime}(W)=L_{\theta}\left(\mathbb{D}(W), \mathfrak{D}^{\prime}(V)\right) .
$$

To each kernel $K_{x, y}$ there corresponds a one-to-one mapping

$$
K_{x, y} \in \mathfrak{D}^{\prime}(V \times W) \rightarrow L_{K} \in \mathscr{D}^{\prime}(V) \hat{\otimes} \mathfrak{D}^{\prime}(W) .
$$

Thus $\mathscr{D}^{\prime}(V \times W)$ can be identified with a subspace of $\mathscr{D}^{\prime}(V) \hat{\otimes} \mathscr{D}^{\prime}(W)$.

The kernel theorem of L. Schwartz [13] asserts that every linear continuous mapping from $D(W)$ into $D^{\prime}(V)$ is defined by a unique kernel. In other words $\mathbb{D}^{\prime}(V \times W)$ can be identified algebraically and topologically with $\mathbb{D}^{\prime}(V) \hat{\otimes} \mathbb{D}^{\prime}(W)$.

DeFINITION 2. A kernel $K_{x, y}$ is called semi-regular in $y$ if the mapping $L_{K}: D(W) \rightarrow D^{\prime}(V)$ can be extended to a linear continuous mapping from $\mathcal{E}^{\prime}(W)$ into $D^{\prime}(V)$.

It is easy to show that $\mathscr{D}^{\prime}(V) \hat{\otimes} \mathcal{E}(W)$ is the subspace of the kernels which are semi-regular in $y$.

Definition 3. A kernel $K_{x, y}$ is called semi-regular in $x$ if the mapping $L_{K}: \mathfrak{D}(W) \rightarrow \mathbb{D}^{\prime}(V)$ maps $\mathfrak{D}(W)$ into $\mathcal{E}(V)$. 
It follows by the closed graph theorem [8, p. 271, Theorem 2] that $L_{K}$ is a continuous mapping from $D(W)$ into $\mathcal{E}(V)$, for, $L_{\boldsymbol{K}}$ being a continuous mapping from $D(W)$ into $D^{\prime}(V)$, its graph is closed in $D(W) \times D^{\prime}(V)$ and, a fortiori, in $\mathscr{D}(W) \times \mathcal{E}(V)$. The topological tensor product $\mathcal{E}(V) \hat{\otimes} \mathscr{D}^{\prime}(W)$ is the space of all the kernels which are semi-regular in $x$.

Definition 4. A kernel $K_{x, y}$ is called regular if it is semi-regular in $x$ and in $y$.

The space of regular kernels coincides with the intersection of

$$
\mathcal{E}(V) \hat{\otimes} D^{\prime}(W) \text { and } D^{\prime}(V) \hat{\otimes} \mathcal{E}(W),
$$

which contains, obviously, the space $\varepsilon(V \times W)=\varepsilon(V) \hat{\otimes} \varepsilon(W)$.

Definition 5. Suppose $V=W$. A kernel $K_{x, y}$ is called very regular if:

(1) $K_{x, y}$ is semi-regular in $y$;

(2) for each $T \in \mathcal{E}^{\prime}(V), L_{K}(T)$ is an infinitely differentiable function on every open set of $V$ on which $T$ is infinitely differentiable.

The following theorem is well known [10, $2^{\mathrm{e}}$ edition, p. 139].

THEOREM 1. A kernel $K_{x, y}$ is very regular if and only if it is regular and $K_{x, y}$ is an infinitely differentiable function in the complement of the diagonal of $V \times V$.

In $\S I I I$, we discuss the analogous problem for analytic kernels.

\section{The SPACE OF REAL ANALYTIC FUnCtions DEFINED ON A COMPACT SUBSET OF $R^{n}$}

Let $K$ be a compact subset of $R^{n}, U$ an open neighborhood of $K$ in $C^{n}$. To each complex analytic function defined on $U$ there corresponds a real analytic function defined on the open neighborhood $U \cap R^{n}$ of $K$ and, conversely, each real analytic function defined on an open neighborhood of $K$ in $R^{n}$ can be extended to a complex analytic function defined on an open neighborhood of $K$ in $C^{n}$.

Let us consider the family of vector spaces $\mathcal{H}(U)$ of complex analytic functions defined on $U$, when $U$ runs through all open neighborhoods of $K$ in $C^{n}$. We say that $f \in \mathcal{F C}(U)$ is equivalent to $g \in \mathcal{F C}(V)$ if and only if $f$ coincides with $g$ on $U \cap V$ and we denote by $a(K)$ the vector space of the classes of equivalent functions. The elements of $a(K)$ will be called analytic functions on $K$ and we denote by $u$ the natural mappings of $\mathcal{F}(U)$ into $a(K)$.

We define on $a(K)$ the inductive limit topology of the spaces $\mathcal{F}(U)$ (we suppose defined on $\mathcal{H C}(U)$ the topology of uniform convergence on compact subsets of $U$ ) by the mappings $u$. It is easy to see that this topology can be obtained by taking an increasing sequence of spaces $\mathfrak{F C}\left(U_{j}\right)$ with the mappings $u_{j}$, where $\left(U_{j}\right)_{j=1,2}, \ldots$ is a decreasing sequence of open neighborhoods of $K$ on $C^{n}$, such that, if $U$ is an open neighborhood of $K$, there exists a $j$ such that $U \supset U_{j}$. 
We can also suppose that each of the connected components of $U_{j}$ contains at least a point of $K$ and in this case the mapping $u_{j}$ will be one to one. Finally, since $K$ is compact, we can take the $U_{j}$ to be relatively compact open sets of $C^{n}$. In this case the inclusion mapping $v_{j}$ from $\mathfrak{H}\left(U_{j}\right)$ into $\mathfrak{C}\left(U_{j+1}\right)$ is a compact mapping.

Let $W_{j}$ be a zero neighborhood in $\mathfrak{K C}\left(U_{j}\right)$ such that $v_{j}\left(W_{j}\right)$ is a relatively compact subset of $\mathfrak{K}\left(U_{j+1}\right)$; let $A_{j+1}$ be the convex circled closed hull of $v_{j}\left(W_{j}\right)$ and $B_{j+1}$ the vector subspace of $\mathfrak{H C}\left(U_{j+1}\right)$ spanned by $A_{j+1}$. With the norm defined by $A_{j+1}$ (see $\left.\S I .1\right), B_{j+1}$ will be a Banach subspace of $\mathfrak{K C}\left(U_{j+1}\right)$. One can show easily that the topology of $a(K)$ defined above coincides with the inductive limit topology of $B_{j}$ by the mappings $u_{j}$.

An important property of $a(K)$ proved by Köthe [9] is that $Q(K)$ is complete. The following theorem, a particular case of a more general one proved by Grothendieck [8, p. 268, Theorem 1] gives a characterization of the bounded subsets of $a(K)$.

Theorem 1. If $A$ is a bounded subset of $Q(K)$, there exists an index $k$ such that $A$ is contained in the image of $\mathcal{H}\left(U_{k}\right)$ by $u_{k}$ and $u_{k}^{-1}(A)$ is bounded in $\mathfrak{F}\left(U_{k}\right)$.

Proof. We may suppose without loss of generality that $A$ is a complete convex circled bounded set and then consider the Banach space $E_{A}$ (see $\S$ I.1). Let us denote by $\phi$ the identity mapping from $E_{A}$ into $Q(K)$.

If there exists a $k$ such that $E_{A} \subset u_{k}\left(\mathfrak{F C}\left(U_{k}\right)\right)$, then by the closed graph theorem [8, p. 271, Theorem 2], $\phi$ which is a continuous mapping from $E_{\boldsymbol{A}}$ into $Q(K)$ will be a continuous mapping from $E_{A}$ into $u_{k}\left(\mathfrak{F}\left(U_{k}\right)\right)$ with the image topology defined by $u_{k}$. Hence $A$ will be a bounded subset of $u_{k}\left(\mathfrak{C}\left(U_{k}\right)\right)$.

Thus, we have to prove that such a $k$ exists. First of all, let

$$
E_{j}=E_{A} \cap u_{j}\left(\mathfrak{F}\left(U_{j}\right)\right) \text {. }
$$

Since $a(K)=U_{j} u_{j}\left(\mathfrak{C}\left(U_{j}\right)\right)$, it follows that $E_{A}=\cup_{j} E_{j}$ and since $E_{A}$ is a Banach space, it follows that there exists an $E_{k}$ which is of second category. Let, now,

$$
G_{j}=\left\{(f, g) \in E_{A} \times \mathfrak{C}\left(U_{j}\right): \phi(f)=u_{j}(g)\right\}
$$

and $p_{j}$ be the projection of $E_{A} \times \mathfrak{K C}\left(U_{j}\right)$ on $E_{A}$; we have $p_{j}\left(G_{j}\right)=E_{j}$. We know that $G_{j}$ and $E_{A}$ are (F) -spaces. By the Banach homomorphism theorem, it follows that if $p_{j}\left(G_{j}\right)=E_{j}$ is distinct from $E_{A}$, then $E_{j}$ is of first category. But, since for some $k, E_{k}$ is of second category, then $E_{k}$ must equal $E_{\boldsymbol{A}}$ and $E_{\boldsymbol{A}}$ is contained in $u_{k}\left(\mathfrak{H}\left(U_{k}\right)\right)$.

Corollary. $a(K)$ is a Montel space.

Proof. If $A$ is a bounded subset in $Q(K)$, then by the preceding theorem, there exists an index $k$ such that $u_{k}^{-1}(A)$ is bounded in $\mathfrak{F C}\left(U_{k}\right)$. Since $\mathfrak{F C}\left(U_{k}\right)$ is a Montel space, then $u_{k}^{-1}(A)$ will be relatively compact, so $A$ will be relatively compact in $Q(K)$. 
Since $a(K)$ is the inductive limit of a sequence of nuclear spaces, $a(K)$ is itself a nuclear space [7, Chapter II, p. 48, Corollary 1] and, hence, $a(K)$ verifies the approximation property. It follows from the remarks of $\$ I .2$ that if $E$ is a complete topological vector space, then

$$
\hat{Q}(K) \hat{\otimes} E=L_{e}\left(E_{c}^{\prime}, a(K)\right) .
$$

If, in particular, $E=a(L)$, where $L$ is a compact subset in $R^{m}$, then the topological tensor product $a(K) \hat{\otimes} Q(L)$, can be interpreted as a space of continuous linear mappings, more precisely, as $L_{e}\left(Q^{\prime}(L), Q(K)\right)$.

Let $K$ (resp. $L$ ) be a compact subset of $R^{n}$ (resp. $R^{m}$ ) and $a(K \times L)$ be the space of classes of complex analytic functions defined on open neighborhoods of $K \times L$ in $C^{n} \times C^{m}$ with the inductive limit topology. Since $\mathfrak{H}(U \times V)$ $=\mathfrak{H}(U) \hat{\otimes} \mathfrak{H}(V)(\S I .2)$, the topology of $\mathscr{Q}(K \times L)$ is the inductive limit topology of $\mathfrak{H}(U) \hat{\otimes} \mathfrak{H}(V)$ by $u \hat{\otimes} v$, where $u$ (resp. $v$ ) is the restriction mapping from $\mathcal{H}(U)$ (resp. $\mathcal{H}(V)$ ) into $Q(K)$ (resp. $Q(L)$ ). As we have remarked, this topology can be obtained by taking a sequence $\mathfrak{H C}\left(U_{i}\right) \hat{\otimes} \mathfrak{H C}\left(V_{i}\right)$ of spaces and the corresponding mappings $u_{i} \hat{\otimes} v_{i}$, where $\left(U_{i} \times V_{i}\right)_{i=1,2, \ldots}$ is a fundamental decreasing sequence of open neighborhoods of $K \times L$ in $C^{n} \times C^{m}$.

It is easy to see that the tensor product $Q(K) \otimes a(L)$ is a dense subspace, of $a(K \times L)$. On $a(K) \otimes a(L)$ we have defined two topologies: the $\pi$-topology, and the induced topology of $a(K \times L)$. We want to prove that these two topologies coincide.

THEOREM 2. On $Q(K) \times a(L)$ the $\pi$-topology coincides with the inductive limit topology of $\mathfrak{H C}\left(U_{i}\right) \hat{\otimes} \mathcal{H C}\left(V_{i}\right)$ by $u_{i} \hat{\otimes} v_{i}$.

Proof. It suffices to show that the dual spaces of $Q(K) \otimes Q(L)$ with the $\pi$-topology and the inductive limit topology are the same with the same equicontinuous sets. As we know ( $(I .2)$, the dual space of $a(K) \otimes_{\pi} a(L)$ is the space $B(a(K), a(L))$ of all continuous bilinear functionals on $a(K) \times a(L)$. If $M$ is an equicontinuous set of $B(a(K), a(L))$, there exists, by definition, a neighborhood $W$ (resp. $W^{\prime}$ ) of the origin in $a(K)$ (resp. $a(L)$ ) such that $|u(f, g)| \leqq 1$ for all $f \in W, g \in W^{\prime}$ and $u \in M$. But, for all $i, u_{i}^{-1}(W)$ (resp. $\left.v_{i}^{-1}\left(W^{\prime}\right)\right)$ is a zero neighborhood in $\mathfrak{H C}\left(U_{i}\right)$ (resp. $\mathfrak{F C}\left(V_{i}\right)$ ). Then, we have: $\left|u\left(u_{i}(\bar{f}), v_{i}(\bar{g})\right)\right| \leqq 1$ for all $\bar{f} \in u_{i}^{-1}(W), \bar{g} \in v_{i}^{-1}\left(W^{\prime}\right)$ and $u \in M$, which means that the set $\left\{u \circ\left(u_{i} \hat{\otimes} v_{i}\right): u \in M\right\}$ is an equicontinuous set of linear functionals on $\mathscr{H}\left(U_{i}\right) \hat{\otimes} \mathfrak{H C}\left(V_{i}\right)$. Hence, $M$ is an equicontinuous set of linear functions with respect to the inductive limit topology of $a(K) \otimes a(L)$.

Let now $M$ be an equicontinuous set of linear functionals on $a(K) \otimes a(L)$ in the inductive limit topology. Since this topology is also the inductive limit topology of the spaces $\mathfrak{F C}\left(U_{i}\right) \hat{\otimes} \mathfrak{H C}\left(V_{j}\right)$ by the mappings $u_{i} \hat{\otimes} v_{j}$, for all $(i, j)$ the set $\left\{u \circ\left(u_{i} \hat{\otimes} v_{j}\right): u \in M\right\}$ is an equicontinuous set of linear forms on $\mathcal{H C}\left(U_{i}\right) \hat{\otimes} \mathcal{H C}\left(V_{j}\right)$. There exist $W_{i}$ and $W_{j}^{\prime}$, respectively, zero neighborhoods in $\mathfrak{H C}\left(U_{i}\right)$ and $\mathfrak{H C}\left(V_{j}\right)$ such that $\left|u\left(u_{i}(\bar{f}), v_{j}(\bar{g})\right)\right| \leqq 1$ for all $\bar{f} \in W_{i}, \bar{g} \in W_{j}$ and 
$u \in M$. Let $W=\Gamma\left(\bigcup_{j} u_{j}\left(W_{j}\right)\right)$ and $W^{\prime}=\Gamma\left(\bigcup_{j} v_{j}\left(W_{j}^{\prime}\right)\right)$ be, respectively, the convex circled hulls of the union of images of $W_{j}$ and $W_{j}^{\prime}$ by $u_{j}$ and $v_{j}$. The sets $W$ and $W^{\prime}$ are zero neighborhoods in $a(K)$ and $a(L)$. Since $f \in W$ and $g \in W^{\prime}$ can be written as

$$
f=\sum_{k=1}^{p} \alpha_{k} u_{j_{k}}\left(\bar{f}_{j_{k}}\right), \quad g=\sum_{k=1}^{p} \beta_{k} v_{j_{k}}\left(\bar{g}_{j_{k}}\right)
$$

where $\sum_{k=1}^{p}\left|\alpha_{k}\right| \leqq 1$ and $\sum_{k=1}^{p}\left|\beta_{k}\right| \leqq 1$, we have:

$$
|u(f, g)| \leqq \sum_{k, l}\left|\alpha_{k}\right|\left|\beta_{l}\right|\left|u\left(u_{j_{k}}\left(\bar{f}_{j_{k}}\right), v_{j_{l}}\left(\bar{g}_{j_{l}}\right)\right)\right| \leqq 1
$$

for all $f \in W, g \in W^{\prime}$ and $u \in M$, which proves that $M$ is an equicontinuous set of $B(a(K), a(L))$; q.e.d.

Let $K$ be a compact subset of $R^{n}$ and $L$ be a compact subset of $R^{m}$; we denote by $Q(K, Q(L))$ the space of classes of complex analytic functions defined on open neighborhoods of $K$ on $C^{n}$, taking their values in $Q(L)$, any two such functions being identified if they coincide on a neighborhood of $K$.

We remark that if $U$ is an open subset of $C^{n}$ and $E$ is a complex topological vector space, then by a vector complex analytic function we mean a function $f$ defined on $U$, taking its values in $E$, which can be represented by a uniformly convergent power series in the neighborhood of each point of $U$.

Analogously, one can define the concept of a complex weakly analytic function. This means a function $f$ defined on $U$, taking its values in $E$, such that, for each $e^{\prime} \in E^{\prime}$, the complex-valued function $f_{\theta^{\prime}}(z)=\left\langle f(z), e^{\prime}\right\rangle$ is analytic on $U$. It is well known that when $E$ is an almost-complete space these two notions coincide.

For our later argument, it is useful to state the following lemma. Its proof is standard and is left to the reader.

LEMMA. Let $E$ be a topological complex vector space and $\left(a_{p} z^{p}\right)$ be a sequence of elements of $E$, where $p=\left(p_{1}, \cdots, p_{n}\right)$ is an n-tuple of nonnegative integers, $z_{p}=z_{1}^{p_{1}} \cdots z_{n}^{p_{n}}$ with $\left(z_{1}, \cdots, z_{n}\right) \in C^{n}$ and $a_{p}=a_{p_{1} \cdots p_{n}} \in E$. Suppose that there exists an $n$-tuple $r=\left(r_{1}, \cdots, r_{n}\right)$ of real numbers $r_{i}>0$ and a complete bounded circled convex set $A$ of $E$ such that $a_{p} r^{p} \in A$ for all $p$. Under these assumptions it follows that the power series $\sum_{p} a_{p} z^{p}$ is absolutely convergent in the Banach space $E_{A}$, for all $|z|<r$ and uniformly convergent on all closed subsets of $|z|<r$. $A$ fortiori, the series will be uniformly convergent in the topology of $E$, on all closed subsets of $|z|<r$.

COROLlARY. Let $E$ be an almost-complete topological vector space and $\sum_{p} a_{p} z^{p}$ a power series with values in $E$. Suppose that there exists an $n$-tuple $\beta=\left(\beta_{1}, \cdots, \beta_{n}\right)$ of complex numbers, $\beta_{i} \neq 0$, such that the sequence $\left(a_{p} \beta^{p}\right)$ is bounded in $E$. Then, if $0<r_{i}<\left|\beta_{i}\right|$, the power series $\sum_{p} a_{p} z^{p}$ will be absolutely and uniformly convergent in a suitable Banach space $E_{A} \subset E$, for $|z| \leqq r . A$ fortiori, the series will be uniformly convergent in $E$, for $|z| \leqq r$. 
Proof. The closed circled convex hull $A$ of $\left(a_{p} \beta^{p}\right)$ is bounded since, by hypothesis, the sequence $\left(a_{p} \beta^{p}\right)$ is bounded. Since $E$ is almost-complete, $A$ is complete and we can apply the results of the lemma to the space $E_{\boldsymbol{A}}$.

We now prove the following theorem.

TheOREM 3. The space $a(K, a(L))$ can be identified with the space $a(K \times L)$.

Proof. Let $f \in a(K, a(L))$ and $\bar{f} \in \mathcal{H}(U, a(L))$ be a representative of $f$ defined on the open neighborhood $U$ of $K$. If $z_{0} \in U$ we have

$$
\bar{f}(z)=\sum_{p} a_{p}\left(z-z_{0}\right)^{p}
$$

where $a_{p} \in Q(L)$, the series being uniformly convergent for $\left|z-z_{0}\right| \leqq r$. For all $z$ in this hyper-cylinder the sequence $\left(a_{p}\left(z-z_{0}\right)^{p}\right)$ is bounded in $Q(L)$, so that by Theorem 1 , it follows that there exists an open neighborhood $V$ of $L$ in $C^{n}$ and representatives $\bar{a}_{p} \in \mathcal{F C}(V)$ of $a_{p}$ such that the sequence $\left(\bar{a}_{p}\left(z-z_{0}\right)^{p}\right)$ is bounded in $\operatorname{FC}(V)$. By the corollary of the lemma, the power series $\sum_{p} \bar{a}_{p}\left(z-z_{0}\right)^{p}$ is uniformly convergent in $\operatorname{TC}(V)$ for $\left|z-z_{0}\right| \leqq r_{1}<r$. Since $z_{0}$ is a fixed but arbitrary point in $U$ we conclude that to each $f \in a(K, a(L))$ there corresponds a function $\bar{f}$ of $\mathfrak{H C}(U, \mathcal{H C}(V))$ represented on a neighborhood of a point $z_{0} \in U$ by the series

$$
\sum_{p} \bar{a}_{p}\left(z-z_{0}\right)^{p}
$$

Since we know that $\mathfrak{H C}(U, \mathfrak{H}(V))=\mathfrak{H}(U \times V)$, this function $\bar{f}$ defines an element of $a(K \times L)$. We obtain in this manner a mapping from $a(K, a(L))$ into $a(K \times L)$ and it is easy to see that this mapping is one-to-one and onto; q.e.d.

Summing up our results, we can write the following identifications between the spaces of analytic functions:

$$
a(K \times L)=a(K, a(L))=a(K) \hat{\otimes} a(L)=L_{0}\left(a^{\prime}(L), a(K)\right),
$$

which state for these spaces the same properties as for the spaces of infinitely differentiable functions.

The result of this section can be extended to the spaces of real analytic functions defined on compact subsets of a real analytic manifold by using the notion of complexification of a real analytic manifold [5].

\section{ANALYTIC Distribution KeRnELS}

Let $V$ be a paracompact real analytic manifold of dimension $n, K$ a compact subset of $V$ and $a(K)$ the vector space of analytic function with the inductive limit topology described in the preceding section. Since $a(K)$ is contained in $\mathcal{E}(K)$ and this last space can be identified with a subspace of $D^{\prime}(V)$ (§I.3), $a(K)$ itself can be considered as a space of distributions on $V$. The elements of $\mathfrak{D}^{\prime}(V \times V)$ will be called, as in $\$ I .3$, distribution kernels. 
We want to define and study properties of analytic kernels like those of the very regular kernels.

Definition 1. A kernel $K_{x, y}$ is called analytic in $x$ (resp. $y$ ) if the following two conditions are verified: (1) $K_{x, y}$ is semi-regular in $y$ (resp. $x$ ); (2) for all $T \in \mathcal{E}^{\prime}(V), L_{K}(T)$ (resp. ${ }^{t} L_{K}(T)$ ) is an analytic function on every open subset of $V$, on which the distribution $T$ is an analytic function.

Definition 2. A kernel $K_{x, y}$ is called analytic if it is analytic in $x$ and in $y$.

THEOREM 1. If $K_{x, y}$ is an analytic kernelin $x$ (resp. y) then $K_{x, y} \in Q(A) \hat{\otimes} \mathcal{E}(B)$ (resp. $K_{x, y} \in \mathcal{E}(A) \hat{\otimes} Q(B)$ ) for all disjoint compact subsets $A$ and $B$ of $V$.

Proof. Suppose $K_{x, y}$ an analytic kernel in $x, A$ and $B$ two disjoint compact subsets of $V$. If $T \in \mathcal{E}^{\prime}(B)$, then from condition (2) of Definition 1 we have that $L_{K}(T) \in Q(A)$. Since $K_{x, y}$ is a semi-regular kernel in $y, L_{K}$ will be a continuous map from $\mathcal{E}^{\prime}(B)$ into $Q(A)$ with the induced topology of $\mathscr{D}^{\prime}(V)$. It follows that the graph of $L_{K}$ in $\mathcal{E}^{\prime}(B) \times Q(A)$ will be closed. But $\mathcal{E}^{\prime}(B)$ is a bornological space $[8$, p. 320 , Corollary 3$]$ and it is a complete space $[8$, p. 207, Exercise 7]. We may, then, apply the closed graph theorem stated by Grothendieck [8, p. 271, Theorem 2] and conclude that $L_{K}$ is a continuous linear map from $\mathcal{E}^{\prime}(B)$ into $Q(A)$, which means by the remarks of $\S I .2$ that $K_{x, y} \in Q(A) \hat{\otimes} \mathcal{E}(B)$. An analogous proof can be carried through when $K_{x, y}$ is an analytic kernel in $y$ and we conclude in this case that $K_{x, y} \in \mathcal{E}(A) \hat{\otimes} Q(B)$.

CoRollary. If $K_{x, y}$ is an analytic kernel, then $K_{x, y}$ is a very regular kernel.

Proof. Since $K_{x, y}$ is a regular kernel, all we have to prove is that $K_{x, y}$ is an infinitely differentiable function in the complement of the diagonal of $V \times V(\S I .3$, Theorem 1). With the same argument as in the preceding theorem we can prove that $L_{K}$ is a continuous linear map from $\mathcal{E}^{\prime}(B)$ into $\mathcal{E}(A)$, in other words that $K_{x, y} \in \mathcal{E}(A) \hat{\otimes} \mathcal{E}(B)=\varepsilon(A \times B)$.

$K_{x, y}$ being an analytic kernel, we remark that, if $x_{0}, y_{0} \in V, x_{0} \neq y_{0}$, it follows from Theorem 1 that $L_{K}\left(\delta_{\left(y_{0}\right)}\right)=K_{x, y_{0}}$ is an analytic function in $x_{0}$ and, also, that $K_{x_{0}, y}$ is an analytic function in $y_{0}$. We conclude from this fact that $K_{x, y}$ is an analytic function in each variable separately, outside the diagonal of $V \times V$. We have, in fact, the following more precise result.

TheORem 2 [2]. If $K_{x, y}$ is an analytic kernel, then $K_{x, y}$ is an analytic function outside the diagonal of $V \times V$.

Proof. Since $K_{x, y} \in[Q(A) \hat{\otimes} \mathcal{E}(B)] \cap[\mathcal{E}(B) \hat{\otimes} a(A)]$ for all disjoint compact subsets of $V$, and since $Q(A) \hat{\otimes} \varepsilon(B)=\mathcal{E}(B, a(A))$ and $\mathcal{E}(A) \hat{\otimes} Q(B)$ $=\mathcal{E}(A, Q(B))(\S I .2)$, the sets $M=\left\{K_{\cdot, y}: y \in B\right\}$ and $N=\left\{K_{x, .}: x \in A\right\}$ are bounded sets in $Q(A)$ and $Q(B)$, respectively, because $A$ and $B$ are compact. There exist, by Theorem 1 of $\S I$, two open neighborhoods $U_{1}$ and $U_{2}$ of $A$ and $B$, respectively, in a fixed complexification of $V$, such that the functions 
of $M$ and $N$ can be extended to complex analytic functions on $U_{1}$ and $U_{2}$, and the sets so obtained are bounded in $\mathfrak{H C}\left(U_{1}\right)$ and $\mathfrak{H C}\left(U_{2}\right)$, respectively. There exists, then, a positive constant $C$ such that $\left|K_{z, y}\right| \leqq C$ for all $z \in U_{1}$ and $y \in B$ and $\left|K_{x, w}\right| \leqq C$ for all $x \in A$ and $w \in U_{2}$.

Under these conditions, Browder [4] has shown that $K_{x, y}$ is an analytic function in both variables. (Essentially, this result states that $[a(A) \hat{\otimes} \mathcal{E}(B)]$ $\cap[\mathcal{E}(A) \hat{\otimes} Q(B)]=Q(A \times B))$.

We give now a partial converse of Theorem 1 of Section I.

THEOREM 3. Let $K_{x, y}$ be a kernel verifying the following conditions: (1) $K_{x, y}$ is semi-regular in $y$ (resp. $x$ ); (2) for all disjoint compact subsets $A$ and $B$ of $V, K_{x, y} \in Q(A) \hat{\otimes} \mathcal{E}(B) \quad$ (resp. $K_{x, y} \in \mathcal{E}(A) \hat{\otimes} Q(B)$ ); (3) if $g \in \mathcal{D}(V), L_{K}(g)$ (resp. ' ${ }^{b} L_{K}(g)$ ) is an analytic function on every open subset of $V$, where $g$ is analytic.

Then $K_{x, y}$ is an analytic kernel in $x$ (resp. $y$ ).

Proof. Suppose $K_{x, y}$ is a kernel, semi-regular in $y$ and verifying the analytic conditions (2) and (3) of the theorem in the $x$ variable. We have to prove condition (2) of Definition 1. Let $T \in \mathcal{E}^{\prime}(V)$ be analytic on an open subset $O$ of $V ; \omega$ a relatively compact open subset of $O$ such that $\bar{\omega} \subset O$ and $\alpha \in \mathfrak{D}(O)$ equal to 1 on an open neighborhood $W$ of $\bar{\omega}$. We have

$$
T=\alpha T+(1-\alpha) T .
$$

Since $\alpha T$ is an infinitely differentiable function with compact support, which is analytic in $\omega$, it follows from (3) that $L_{K}(\alpha T)$ is analytic in $\omega$. On the other hand, $(1-\alpha) T$ is a distribution with compact support $k$ contained in the intersection of the support of $T$ with the complement of $W$; hence $k$ is disjoint of $\bar{\omega}$. Let us set $A=\bar{\omega}$ and $B=k$. By condition (2), above, we have $K_{x, y} \in Q(A) \hat{\otimes} \&(B)$. It follows, then, that $L_{K}((1-\alpha) T) \in Q(A)$, hence it is analytic in $\omega$. We conclude that $L_{K}(T)$ is analytic in $\omega$. Since $\omega$ is an arbitrary but relatively compact subset of $O, L_{K}(T)$ is analytic in $O$. The same proof holds when $K_{x, y}$ is a kernel, semi-regular in $x$ and verifying the analytic conditions above in the $y$ variable.

COROLLARY. Let $K_{x, y}$ be a kernel satisfying the following three conditions:

(1) $K_{x, y}$ is regular;

(2) $K_{x, y}$ is an analytic function on the complement of the diagonal on $V \times V$;

(3) if $g \in D(V)$, then $L_{K}(g)$ and ${ }^{'} L_{K}(g)$ are analytic functions on every open subset on which $g$ is analytic.

Then $K_{x, y}$ is an analytic kernel.

The corollary establishes in the analytic case, the analogue of the sufficient condition of Theorem 1 in $\S \mathrm{I}$. We remark that, to obtain this parallel conclusion, we need to impose another condition, namely condition (3), which in the infinitely differentiable case is contained in the assumption of 
regularity of the kernel. The following example shows that condition (3) is essential.

Suppose $V=R^{n}$ and take $I_{x, y}$ to be the kernel

$$
\left\langle I_{x, y}, \Phi(x, y)\right\rangle=\int_{R^{n}} \Phi(t, t) d t
$$

for all $\Phi \in \mathscr{D}\left(R^{n} \times R^{n}\right)$. $I_{x, y}$ represents the unit mass on the diagonal. Since its support is contained in the diagonal, $I_{x, y}$ can be identified to the zero function off the diagonal. Let $\alpha(x)$ be an infinitely differentiable function defined on $R^{n}$ and let $K_{x, y}=\alpha(x) I_{x, y}$ be the kernel defined by

$$
\left\langle K_{x, y}, \Phi(x, y)\right\rangle=\int_{R^{n}} \alpha(x) \Phi(x, x) d x
$$

for all $\Phi \in \mathscr{D}\left(R^{n} \times R^{n}\right)$. We have, obviously, $L_{K}(g)=\alpha \cdot g$ for all $g \in \mathscr{D}\left(R^{n}\right)$ and we see that condition (3) does not hold if $\alpha$ is not analytic.

\section{BIBLIOGRAPHY}

1. J. Barros Neto, Alguns tipos de nucleos-distribuiçōes, Thesis, Universidade de São Paulo, 1960.

2. J. Barros Neto and F. E. Browder, The analyticity of kernels, Canad. J. Math., to appear.

3. N. Bourbaki, Espaces vectoriels topologiques, tomes 1 et 2, Paris, Hermann \& Cie, 1953.

4. F. E. Browder, Real analytic functions on product spaces and separate analyticity, Canad. J. Math. to appear.

5. F. Bruhat and $\mathrm{H}$. Whitney, Quelques proprietés fondamentales des ensembles analytiques reels, Comment. Math. Helv. vol. 33 (1959) pp. 132-160.

6. J. Dieudonné and L. Schwartz, La dualité dans les espaces (F) et (\&F), Ann. Inst. Fourier Grenoble Vol. 1 (1949) pp. 61-101.

7. A. Grothendieck, Produits tensoriels topologiques et espaces nucléaires, Memoirs Amer. Math. Soc. no. 16 (1955); erratum, Ann. Inst. Fourier Grenoble vol. 6 (1955/56) pp. 117-120.

8. - Espaces vectoriels topologiques, São Paulo, 1954.

9. G. Köthe, Über die Vollständigkeit einer Klasse lokalkonvexer Räume, Math. Z. vol. 52 (1950) pp. 627-630.

10. L. Schwartz, Théorie des distributions, tomes I, II, Actualités Sci. Ind. no. 1091, 1122, Paris, Hermann \& Cie, 1950, 1951.

11. - Theorie des noyaux, Proceedings of the International Congress of Mathematicians, 1950, American Mathematical Society, 1952.

12. - Produits tensoriels topologiques, Séminaire, Paris, 1953/54.

13. - Theorie des distributions a valeurs vectoriels, Ann. Inst. Fourier Grenoble vol. 7 (1957) p. 1.

YALE UNIVERSITY,

New Haven, Connecticut

Institute for PURE AND APPLied Mathematics,

RIO DE JANEIRO, BRAZIT. 